\title{
DILATIONS OF SEMIGROUP CROSSED PRODUCTS AS CROSSED PRODUCTS OF DILATIONS
}

\author{
NADIA S. LARSEN AND XIN LI \\ (Communicated by Marius Junge)
}

\begin{abstract}
Laca constructed a minimal automorphic dilation for every semigroup dynamical system arising from an action of an Ore semigroup by injective endomorphisms of a unital $C^{*}$-algebra. Here we show that the semigroup crossed product with its action by inner endomorphisms given by the implementing isometries has as minimal automorphic dilation the group crossed product of the original dilation. Applications include recent examples studied by Cuntz and the second-named author.
\end{abstract}

\section{INTRODUCTION}

Starting from the work of Cuntz in [Cu1, the theory of semigroup crossed products has consolidated itself as a rich source of many new and interesting examples of $C^{*}$-algebras. In Cu1, semigroup crossed products appear as corners in ordinary group crossed products. Later, in [Sta, a definition of semigroup crossed products was introduced in terms of a universal property for a covariance relation relating representations of the $C^{*}$-algebra and representations of the semigroup. Much of the subsequent theory developed along this path; see for example Mur and La-Ra1. However, the corner representation in an ordinary group crossed product played an important role; see Mur. Laca extended the work of Murphy to a large class of not necessarily abelian semigroups, the Ore (right-reversible) semigroups which act by injective endomorphisms of a unital $C^{*}$-algebra; see [La].

Recent new examples of $C^{*}$-algebras arising in number theory and in a purely algebraic context turned out to be instances of semigroup crossed products; see $\mathrm{Cu} 2$, $\mathrm{Cu}-\mathrm{Li} 1$, $\mathrm{Cu}-\mathrm{Li} 2$ and [Li]. From these semigroup crossed products isomorphisms emerge involving ordinary group crossed products by affine-type groups. These isomorphisms play an important role in the $K$-theory computations.

The goal of this note is to establish a general result about the minimal automorphic dilation of a certain type of semigroup dynamical system. Given a semigroup dynamical system, one can first dilate the dynamical system to the effect that the

Received by the editors October 6, 2010.

2010 Mathematics Subject Classification. Primary 46L55.

The first-named author thanks J. Cuntz and S. Echterhoff for their kind hospitality during a sabbatical visit at Westfälische Wilhelms-Universität Münster in October 2009, where this research was initiated.

The second-named author thanks the operator algebra group in Oslo for a nice visit at the University of Oslo.

This research was supported by the Research Council of Norway and the Deutsche Forschungsgemeinschaft. 
endomorphisms become automorphisms and then form the crossed product (by a group), or one can first form the semigroup crossed product and then dilate the resulting $C^{*}$-algebra. Our main result (Theorem 1.3) says that the outcome is the same.

We were led to this result by certain isomorphisms established in $\mathrm{Cu} 2$ and [Cu-Li1] (see Section 2). They turn out to be special cases of our main theorem. Therefore, this note gives a unified treatment of these phenomena.

\section{Dilations of Semigroup CRossed products}

Let $A$ be a unital $C^{*}$-algebra and $P$ a discrete semigroup with an identity element. We always assume that our semigroups are discrete and have an identity element. Let $\alpha$ be an action of $P$ on $A$, so $\alpha$ is a semigroup homomorphism $P \rightarrow$ End $(A)$ preserving the identity elements. We associate the semigroup crossed product $A \stackrel{e}{\rtimes_{\alpha}} P$ with the semigroup $C^{*}$-dynamical system $(A, P, \alpha)$. The symbol $\stackrel{e}{\rtimes}$ stands for "semigroup crossed product by endomorphisms". The $C^{*}$-algebra $A \stackrel{e}{\rtimes_{\alpha}} P$ is universal for covariant representations of $(A, P, \alpha)$ : these are pairs $(\pi, W)$ consisting of a nondegenerate representation $\pi$ of $A$ on a Hilbert space $H$ and a homomorphism $W$ from $P$ into the semigroup of isometries on $H$ such that the covariance condition $\pi\left(\alpha_{p}(a)\right)=W_{p} \pi(a) W_{p}^{*}$ is satisfied for all $a \in A$ and $p \in P$.

The crossed product $A \stackrel{e}{\rtimes}_{\alpha} P$ is generated as a $C^{*}$-algebra by a universal covariant representation $\left(i_{A}, i_{P}\right)$; see for example [Sta, Mur, La-Ra1. One can argue that the idea behind the definition of $A \stackrel{e}{\rtimes_{\alpha}} P$ is to enlarge $A$ by adding isometries $w_{p}=i_{P}(p)$ for all $p \in P$ so that the action of $\alpha$ becomes inner, in the sense that $\alpha_{p}(\cdot)=w_{p} \cdot w_{p}^{*}$ for all $p \in P$. In general, it may happen that $A \stackrel{e}{\rtimes_{\alpha}} P$ is zero; see e.g. [Sta, Example 2.1(a)].

In this note we will be concerned with actions of Ore semigroups. Recall that a (right-reversible) Ore semigroup is a cancellative semigroup $P$ such that $P s \cap P t \neq \emptyset$ for all $s, t \in P$. There is a similar notion of left-reversible Ore semigroups. A cancellative semigroup is Ore precisely when it can be embedded in a group $G$ in such a way that $G=P^{-1} P . G$ is called the enveloping group of $P$ and is determined uniquely up to isomorphism by $P$; cf. Cli-Pre, Theorems 1.23, 1.24, 1.25]. The semigroup structure of $P$ induces a partial pre-order on $P$ (and also on $G$ ) given by $p \leq r$ if $r \in P p$.

Let $\alpha$ be an action of an Ore semigroup $P$ on a unital $C^{*}$-algebra $A$. If there is a nontrivial covariant representation for $(A, P, \alpha)$, then $A \stackrel{e}{\rtimes}_{\alpha} P$ is nonzero by e.g. La-Ra1, and due to the right-reversibility of $P$ the crossed product $A \stackrel{e}{\rtimes_{\alpha}} P$ is the closed span of the monomials $i_{P}(p)^{*} i_{A}(a) i_{P}(r)$ for $a \in A$ and $p, r \in P$; see [La].

Fix a semigroup dynamical system $(A, P, \alpha)$ with $P$ an Ore semigroup and $\alpha$ an action of $P$ by endomorphisms $\alpha_{p}$ which are injective for all $p \in P$. Laca used dilation theory for projective representations of semigroups to show that the existence of a nontrivial covariant representation for this kind of $(A, P, \alpha)$ is automatic. Indeed, if $(A, P, \alpha)$ is a semigroup dynamical system with $P$ an Ore semigroup and $\alpha$ an action by injective endomorphisms, let $G=P^{-1} P$ be the enveloping group of $P$, and recall that $\left[\mathrm{La}\right.$, Theorem 2.1 ] shows that there exist an ordinary $C^{*}$-dynamical 
system $(B, G, \beta)$ and an embedding $\iota: A \hookrightarrow B$ such that

$$
\beta_{p} \circ \iota=\iota \circ \alpha_{p} \quad \text { for all } p \in P
$$

and

$$
\overline{\bigcup_{p \in P} \beta_{p}^{-1}(\iota(A))}=B .
$$

The triple $(B, G, \beta)$ is the minimal automorphic dilation of $(A, P, \alpha)$ and is unique up to isomorphism. The identities in (11) express the dilation phenomena, and condition (2) is the minimality. Laca's other main result shows that $A \stackrel{e}{\rtimes}_{\alpha} P$ can be realised as a full corner of the ordinary crossed product $B \rtimes_{\beta} G$; cf. [La, Theorem 2.4].

Remark 1.1. A model for $B$ is given as follows (see the proof of [La, Theorem 2.1]): Take the direct limit over the directed set $P$ of copies $A_{p}$ of $A$ for all $p \in P$ with connecting homomorphisms $\alpha_{r}^{p}: A_{p} \rightarrow A_{r}$ given by $\alpha_{r}^{p}:=\alpha_{r p^{-1}}$ when $p \leq r$. Then there is an action $\alpha_{\infty}$ of $G$ on $A_{\infty, \alpha}=\lim _{P}\left\{A ; \alpha_{r}^{p}\right\}_{r \leq p}$ such that $\left(A_{\infty, \alpha}, G, \alpha_{\infty}\right)$ and the embedding $\iota^{1}: A_{e} \rightarrow A_{\infty, \alpha}$ satisfy (11) and (2). Here $e$ is the identity element of $P$.

Let $1_{A}$ denote the unit of $A$, let $w_{p}=i_{P}(p)$ for $p \in P$ and let $u_{g}, g \in G$, be the unitaries in the multiplier algebra of $B \rtimes_{\beta} G$ which implement $\beta$. The inclusion $\iota: A \hookrightarrow B$ induces an inclusion $A \stackrel{e}{\rtimes}_{\alpha} P \hookrightarrow B \rtimes_{\beta} G$, also denoted $\iota$, with the property that

$$
\iota\left(w_{p}\right)=u_{p} \iota\left(1_{A}\right)
$$

for $p \in P$; see [La, Theorem 2.4]. We note the following.

Lemma 1.2. The net $\left(u_{p}^{*} \iota\left(1_{A}\right) u_{p}\right)_{p \in P}$ is an approximate unit in $B$.

Proof. This is an immediate consequence of the fact that $u_{p}^{*} \cdot u_{p}=\beta_{p}^{-1}(\cdot)$ for all $p$ in $P$ and that $\bigcup_{p \in P} u_{p}^{*} \iota(A) u_{p}$ is dense in $B$ by (2).

Next we notice that we can form a new semigroup dynamical system with injective endomorphisms by letting $\phi$ be the action of $P$ on $A \stackrel{e}{\rtimes_{\alpha}} P$ given by $\phi_{p}:=\operatorname{Ad} w_{p}$ for $p \in P$. Moreover, let $\psi$ be the action of $G$ on $B \rtimes_{\beta} G$ given by $\psi_{g}:=\operatorname{Ad} u_{g}$ for $g$ in $G$.

The main thrust of this note is that the dilation of the new system $\left(A \stackrel{e}{\rtimes_{\alpha}}\right.$ $P, P, \phi)$ is the crossed product $B \rtimes_{\beta} G$. This result was suggested by very recent examples of actions arising in number-theoretic and purely algebraic contexts; see $\mathrm{Cu} 2, \mathrm{Cu}-\mathrm{Li1}, \mathrm{Cu}-\mathrm{Li} 2$.

Theorem 1.3. The embedding $\iota: A \stackrel{e}{\rtimes_{\alpha}} P \hookrightarrow B \rtimes_{\beta} G$ and the dynamical system $\left(B \rtimes_{\beta} G, G, \psi\right)$ form the minimal automorphic dilation of $\left(A \stackrel{e}{\rtimes}_{\alpha} P, P, \phi\right)$.

Proof. By [La, Theorem 2.1] we have to show the dilation phenomena and the minimality. That $\psi_{p}$ dilates $\phi_{p}$ for every $p$ in $P$ in the sense of (1) follows from (3).

To show that $\left(B \rtimes_{\beta} G, G, \psi\right)$ is minimal, we need to show that any $y \in B \rtimes_{\beta} G$ can be approximated from $\bigcup_{p \in P} u_{p}^{*}\left(\iota\left(A \stackrel{e}{\rtimes_{\alpha}} P\right)\right) u_{p}$. It suffices to prove this claim 
for $y=b u_{g}$ for $b$ in $B$ and $g$ in $G$ because elements of this form span a dense subset of $B \rtimes_{\beta} G$. So fix $b$ and $g$. By (2), we can find a net $\left(a_{p}\right)_{p \in P}$ in $A$ such that

$$
b=\lim _{p} u_{p}^{*} \iota\left(a_{p}\right) u_{p} .
$$

Since $G=P^{-1} P$, we can find for all $p$ in $P$ elements $q_{p}$ and $r_{p}$ in $P$ such that $q_{p}^{-1} r_{p}=p g p^{-1}$. Then $u_{p g p^{-1}}=u_{q_{p}^{-1} r_{p}}=u_{q_{p}}^{*} u_{r_{p}}$. Hence, using (3) , we obtain

$$
\begin{aligned}
u_{p}^{*}\left(\iota\left(a_{p} w_{q_{p}}^{*} w_{r_{p}}\right)\right) u_{p} & =u_{p}^{*} \iota\left(a_{p}\right) \iota\left(w_{q_{p}}^{*}\right) \iota\left(w_{r_{p}}\right) u_{p} \\
& =u_{p}^{*} \iota\left(a_{p}\right) u_{q_{p}}^{*} u_{r_{p}} \iota\left(1_{A}\right) u_{p} \\
& =u_{p}^{*} \iota\left(a_{p}\right) u_{p} u_{g} u_{p}^{*} \iota\left(1_{A}\right) u_{p} .
\end{aligned}
$$

Lemma 1.2 and (44) imply that the term in (5) converges to $b u_{g}$. Hence $b u_{g}$ can be approximated from $\bigcup_{p \in P} u_{p}^{*}\left(\iota\left(A \stackrel{e}{\rtimes}_{\alpha} P\right)\right) u_{p}$, as claimed.

Remark 1.4. In other words, we have shown that the $C^{*}$-algebras

$$
B \rtimes_{\beta} G \text { and } \underset{p \in P}{\lim }\left\{A \stackrel{e}{\rtimes}_{\alpha} P ; \phi_{p}\right\}
$$

are canonically isomorphic.

In the cases of interest for our applications the semigroup $P$ has the form $P=$ $H \rtimes_{\eta} M$, where $M$ is a right-reversible Ore semigroup, $H$ is a group and $\eta$ : $M \rightarrow$ End $(H)$ an action of $M$ by injective endomorphisms of $H$. Both $H$ and $M$ can be embedded as subsemigroups in $P$ via $H \ni h \mapsto\left(h, e_{M}\right) \in P$ and $M \ni$ $m \mapsto\left(e_{H}, m\right) \in P$, where $e_{H}$ and $e_{M}$ denote the identity elements in $H$ and $M$, respectively. Since $H$ is a group, the action $\alpha$ of $P$ restricts to an action $\left.\alpha\right|_{H}$ of $H$ by automorphisms (as long as the identity element of $P$ acts as the identity homomorphism). It follows from our assumptions that $P$ is again an Ore semigroup. Let $G$ be the enveloping group of $P$. The equality $\left(-h, e_{M}\right)(h, m)=\left(e_{H}, m\right)$ shows that the semigroup $M$ is a cofinal subset of $P$ (i.e. for any $(h, m) \in P$ there is an element $x \in M$ such that $(h, m) \leq x$ in the right-order on $P$ ). Therefore an inductive limit over $P$ can be viewed as an inductive limit over $M$. Hence Theorem 1.3 implies that the embedding $\iota: A \stackrel{e}{\rtimes_{\alpha}} P \hookrightarrow B \rtimes_{\beta} G$ and the triple $\left(B \rtimes_{\beta} G, M^{-1} M,\left.\psi\right|_{M^{-1} M}\right)$ form the minimal automorphic dilation of the semigroup dynamical system $\left(A \stackrel{e}{\rtimes}_{\alpha} P, M,\left.\phi\right|_{M}\right)$. With the notation of Remark 1.1,

$$
\left(A \stackrel{e}{\rtimes}_{\alpha} P\right)_{\infty,\left.\phi\right|_{M}} \cong B \rtimes_{\beta} G .
$$

Remark 1.5. Note that in the case $P=H \rtimes_{\eta} M$, the condition (2) expressing the minimality of the minimal automorphic dilation $(B, G, \beta)$ takes the following form:

$$
\bigcup_{m \in M} \beta_{\left(e_{H}, m\right)}^{-1}(\iota(A)) \text { is dense in } B .
$$

This follows from cofinality of $M$ in $P$.

The motivating example is that of $\mathbb{Z} \rtimes \mathbb{N}^{\times}$, where the multiplicative semigroup $\mathbb{N}^{\times}$of nonzero natural numbers acts on $\mathbb{Z}$ by multiplication $n \mapsto k n$ for $k \in \mathbb{N}^{\times}$ and $n \in \mathbb{Z}$. It is easily seen that $\mathbb{Z} \rtimes \mathbb{N}^{\times}$is right-reversible. Note that already $\mathbb{N} \rtimes \mathbb{N}^{\times}$is right-reversible, where $\mathbb{N}$ is the additive semigroup of natural numbers with 0 . But $\mathbb{N}^{\times}$is not cofinal in $\mathbb{N} \rtimes \mathbb{N}^{\times}$for the right-order. However, $\mathbb{N} \rtimes \mathbb{N}^{\times}$ is not left-reversible, since for pairs $(l, k),(n, m) \in \mathbb{N} \rtimes \mathbb{N}^{\times}$such that $n-l$ is not 
congruent to $0 \bmod \operatorname{gcd}(k, m)$ we have $(l, k)\left(\mathbb{N} \rtimes \mathbb{N}^{\times}\right) \cap(n, m)\left(\mathbb{N} \rtimes \mathbb{N}^{\times}\right)=\emptyset$; see [La-Ra2, Proposition 2.2] (there it is shown that $\left(\mathbb{Q} \rtimes \mathbb{Q}_{+}^{\times}, \mathbb{N} \rtimes \mathbb{N}^{\times}\right)$is quasi-lattice ordered with respect to its left-order).

\section{Applications}

2.1. The $C^{*}$-algebra $\mathcal{Q}_{\mathbb{N}}$. The first application concerns the $C^{*}$-algebra $\mathcal{Q}_{\mathbb{N}}$ constructed in $\mathrm{Cu} 2$. Recall from $\mathrm{Cu} 2$ that $\mathcal{Q}_{\mathbb{N}}$ is the universal $C^{*}$-algebra generated by isometries $s_{m}$ for $m \in \mathbb{N}^{\times}$and a unitary $u$ subject to the relations $s_{k} s_{m}=s_{k m}$, $s_{m} u=u^{m} s_{m}$ and $\sum_{n=0}^{m-1} u^{n} s_{m} s_{m}^{*} u^{-n}=1$ for $k, m \in \mathbb{N}^{\times}$. Let $P_{\mathbb{N}}$ be the semidirect product $\mathbb{N} \rtimes \mathbb{N}^{\times}$arising from the action of $\mathbb{N}^{\times}$on $\mathbb{N}$ given by $k \cdot n=k n$ for $k \in \mathbb{N}^{\times}$and $n \in \mathbb{N}$. (Note that this $P_{\mathbb{N}}$ is the opposite semigroup of the semigroup of matrices denoted by the same symbol in $\mathrm{Cu} 2$.) The operation in $P_{\mathbb{N}}$ is given by $(l, k)(n, m)=(l+k n, k m)$ for $(l, k),(n, m) \in P_{\mathbb{N}}$.

Let $e_{m}=s_{m} s_{m}^{*}$ denote the range projection of $s_{m}$ for all $m \in \mathbb{N}^{\times}$. It is shown in $\mathrm{Cu} 2$ that the $C^{*}$-algebra $\mathcal{D}$ generated by the projections $u^{n} e_{m} u^{-n}$, where $0 \leq$ $n<m$ for all $m \in \mathbb{N}^{\times}$, is commutative. The semigroup $P_{\mathbb{N}}$ acts on $\mathcal{D}$ by $\alpha_{(n, m)}:=$ $\operatorname{Ad}\left(u^{n} s_{m}\right)$. Let $\left(i_{\mathcal{D}}, i_{P}\right)$ denote the universal covariant pair of the semigroup system $\left(\mathcal{D}, P_{\mathbb{N}}, \alpha\right)$. Let $w_{(n, m)}=i_{P}(n, m)$ for $n \in \mathbb{Z}, m \in \mathbb{N}^{\times}$. It follows that the isometries $w_{(0, m)}$ for $m \in \mathbb{N}^{\times}$and the unitary $w_{(1,1)}$ satisfy the relations defining $\mathcal{Q}_{\mathbb{N}}$. By the universal property of $\mathcal{Q}_{\mathbb{N}}$ there is a homomorphism from $\mathcal{Q}_{\mathbb{N}}$ to $\mathcal{D} \stackrel{e}{\rtimes_{\alpha}} P_{\mathbb{N}}$ sending $u$ to $w_{(1,1)}$ and $s_{m}$ to $w_{(0, m)}$ for all $m \in \mathbb{N}^{\times}$. The algebra $\mathcal{D} \stackrel{e}{\rtimes}_{\alpha} P_{\mathbb{N}}$ is the closed span of monomials of the form $w_{(l, k)}^{*} i_{\mathcal{D}}(f) w_{(n, m)}$ for $f \in \mathcal{D},(l, k),(n, m) \in P_{\mathbb{N}}$. Writing $w_{(n, m)}=w_{(1,1)}^{n} w_{(0, m)}$ and using that $\mathcal{D}$ is generated by projections of the form $u^{n} e_{m} u^{-n}$ show that $\mathcal{D} \stackrel{e}{\rtimes}_{\alpha} P_{\mathbb{N}}$ is generated as a $C^{*}$-algebra by the elements $w_{(1,1)}$ and $w_{(0, m)}$ for $m \in \mathbb{N}^{\times}$. Simplicity of $\mathcal{Q}_{\mathbb{N}}$ implies that the homomorphism defined above is an isomorphism, i.e.

$$
\mathcal{Q}_{\mathbb{N}} \cong \mathcal{D} \stackrel{e}{\rtimes}_{\alpha} P_{\mathbb{N}}
$$

This fact is implicit in $\mathrm{Cu} 2$ and follows from taking $R=\mathbb{Z}$ in $\mathrm{Cu}-\mathrm{Li1}$; see Paragraph 2.2

Since $u$ is a unitary, $\alpha$ extends to an action of $\mathbb{Z} \rtimes \mathbb{N}^{\times}$on $\mathcal{D}$ (also denoted by $\alpha$ ). Moreover, $\mathcal{D} \stackrel{e}{\rtimes}_{\alpha} P_{\mathbb{N}}$ and $\mathcal{D} \stackrel{e}{\rtimes}_{\alpha}\left(\mathbb{Z} \rtimes \mathbb{N}^{\times}\right)$are canonically isomorphic. It is easy to see that the $(a x+b)$-group $P_{\mathbb{Q}}^{+}=\mathbb{Q} \rtimes \mathbb{Q}_{+}^{\times}$is the enveloping group of $\mathbb{Z} \rtimes \mathbb{N}^{\times}$. By Theorem 1.3 as formulated in (6), $\left(\mathcal{D} \stackrel{e}{\rtimes}_{\alpha} P_{\mathbb{N}}\right)_{\infty,\left.\phi\right|_{\mathbb{N} \times}}$ is isomorphic to $\mathcal{D}_{\infty, \alpha} \rtimes_{\beta} P_{\mathbb{Q}}^{+}$.

It is proved in $\mathrm{Cu} 2$ that $\mathcal{D}$ is isomorphic to $C(\hat{\mathbb{Z}})$; the isomorphism sends $u^{n} e_{m} u^{-n}$ to the characteristic function of the set $n+m \hat{\mathbb{Z}}$; see $\mathrm{Cu}$-Li1. Here $\hat{\mathbb{Z}}$ is the profinite completion of the integers. Under this isomorphism the action $\alpha$ of $P_{\mathbb{N}}$ on $\mathcal{D}$ is transformed into the action of $P_{\mathbb{N}}$ on $C(\hat{\mathbb{Z}})$ given by affine transformations,

$$
\alpha_{(n, m)}^{\text {aff }}(f)(x)=\left\{\begin{array}{l}
f\left(m^{-1}(x-n)\right), \quad \text { if } x \in n+m \hat{\mathbb{Z}}, \\
0, \text { otherwise. }
\end{array}\right.
$$

It follows from $\mathrm{Cu} 2$ and $\mathrm{Cu}$-Li1 that $\mathcal{D}_{\infty, \alpha}$ is isomorphic to $C_{0}\left(\mathbb{A}_{f}\right)$, where $\mathbb{A}_{f}$ is the finite adele ring over the rationals. The embedding of $\mathcal{D}$ into $\mathcal{D}_{\infty, \alpha}$ is the canonical embedding $\iota: C(\hat{\mathbb{Z}}) \rightarrow C_{0}\left(\mathbb{A}_{f}\right)$ obtained from cutting down with the projection equal to the characteristic function of $\hat{\mathbb{Z}}$. The action $\beta$ of $P_{\mathbb{Q}}^{+}$extending 
$\alpha^{\text {aff }}$ is the action by affine transformations on $C_{0}\left(\mathbb{A}_{f}\right)$. Taking into account (7), we have established that $C_{0}\left(\mathbb{A}_{f}\right) \rtimes_{\alpha^{\text {aff }}} P_{\mathbb{Q}}^{+}$is isomorphic to the dilation of $\mathcal{Q}_{\mathbb{N}}$ by the endomorphisms $x \mapsto s_{m} x s_{m}^{*}, m \in \mathbb{N}^{\times}$. This dilation is the algebra $\overline{\mathcal{Q}}_{\mathbb{N}}$ from [Cu2], and we have recovered [Cu2, Theorem 6.4].

2.2. $C^{*}$-algebras associated to integral domains. Similarly to the previous example we now outline how to use Theorem 1.3 to recover [Cu-Li1, Theorem 2].

Recall the following from $\mathrm{Cu}-\mathrm{Li1}$ : Suppose that $R$ is an integral domain with set of units strictly contained in $R^{\times}=R \backslash\{0\}$ and such that for each $m \in R^{\times}$ the ideal $(m)$ of $R$ is of finite index in $R$. Then $\mathfrak{A}[R]$ is the universal $C^{*}$-algebra generated by isometries $\left\{s_{m} \mid m \in R^{\times}\right\}$and unitaries $\left\{u^{n} \mid n \in R\right\}$ subject to a number of relations (in the case $R=\mathbb{Z}$ these are the relations defining $\mathcal{Q}_{\mathbb{N}}$ ). The $C^{*}$-algebra $\mathcal{D}[R]:=\overline{\operatorname{span}}\left\{u^{n} e_{m} u^{-n} \mid n \in R, m \in R^{\times}\right\}$is commutative and is isomorphic to the algebra of continuous functions on the profinite completion $\hat{R}$ of $R$.

To apply Theorem 1.3 we need to start with a semigroup crossed product and dilate it to obtain an ordinary group crossed product. Therefore we proceed in the opposite direction as that pursued in [Cu-Li1] and start by identifying $\mathfrak{A}[R]$ with a semigroup crossed product. Indeed, let $P_{R}$ be the right-reversible semigroup $R \rtimes R^{\times}$, where $R^{\times}$acts multiplicatively on the additive group $R$. Let $\alpha_{(l, k)}:=\operatorname{Ad}\left(u^{l} s_{k}\right)$ for $(l, k) \in P_{R}$. Then $\alpha_{(l, k)}\left(u^{n} e_{m} u^{-n}\right)=u^{l+k n} e_{k m} u^{-(l+k n)}$ for all $(l, k),(n, m) \in P_{R}$, and it follows that $\alpha$ is an action of $P_{R}$ by injective endomorphisms of $\mathcal{D}[R]$. If $\left(i_{\mathcal{D}}, i_{P}\right)$ is the universal covariant pair of $\left(\mathcal{D}[R], P_{R}, \alpha\right)$, then $i_{\mathcal{D}}(1)$ is the identity element in the semigroup crossed product and each $w_{(n, m)}:=i_{P}(n, m)$ is an isometry for $(n, m) \in P_{R}$. It follows from our construction that the isometries $w_{(0, m)}$ for $m \in R^{\times}$and the unitaries $w_{(n, 1)}$ for $n \in R$ satisfy the relations defining $\mathfrak{A}[R]$. Moreover, it follows as in Section 2.1 that $w_{(n, 1)}$ and $w_{(0, m)}$ for $n \in R$ and $m \in R^{\times}$ generate $\mathcal{D}[R] \stackrel{e}{\rtimes_{\alpha}} P_{R}$. Then simplicity of $\mathfrak{A}[R]$, established in [Cu-Li1], implies that there is an isomorphism of $\mathfrak{A}[R]$ onto $\mathcal{D}[R] \rtimes_{\alpha} P_{R}$ which carries $s_{m}$ to $w_{(0, m)}$ for $m \in R^{\times}$.

The enveloping group of $P_{R}$ is $P_{Q(R)}$, the $(a x+b)$-group over the quotient field $Q(R)$ of $R$. Theorem 1.3 implies that $(\mathcal{D}[R])_{\infty, \alpha} \rtimes_{\beta} P_{Q(R)}$ is isomorphic to the dilation $\left(\mathcal{D}[R] \stackrel{e}{\rtimes_{\alpha}} P_{R}\right)_{\infty,\left.\phi\right|_{R^{\times}}}$, and so there is an isomorphism

$$
(\mathcal{D}[R])_{\infty, \alpha} \rtimes_{\beta} P_{Q(R)} \cong(\mathfrak{A}[R])_{\infty,\left.\mathrm{Ad}\right|_{R^{\times}}} .
$$

It was shown in $\mathrm{Cu}$-Li1 that $(\mathcal{D}[R])_{\infty, \alpha}$ is isomorphic to $C_{0}(\mathscr{R})$, where $\mathscr{R}$ is the generalised finite adele ring associated with $R$. The $C^{*}$-algebra $(\mathfrak{A}[R])_{\infty,\left.A d\right|_{R} \times}$ is the stabilisation $\mathfrak{A}(R)$ from [Cu-Li1, and (9) recovers therefore the isomorphism of $\mathfrak{A}(R)$ with $C_{0}(\mathscr{R}) \rtimes P_{Q(R)}$. This is [Cu-Li1, Theorem 2].

\section{REFERENCES}

[Cli-Pre] A.H. Clifford and G. B. Preston, The algebraic theory of semigroups - Vol. I, Mathematical Surveys 7, American Mathematical Society, Providence, Rhode Island, 1961. MR 0132791 (24:A2627)

[Cu1] J. Cuntz, Simple $C^{*}$-algebras generated by isometries, Comm. Math. Phys. 57 (1977), no. 2, 173-185. MR0467330 (57:7189)

[Cu2] J. Cuntz, $C^{*}$-algebras associated with the ax + -semigroup over $\mathbb{N}$, in $K$-Theory and noncommutative geometry (Valladolid, 2006), European Math. Soc., 2008, pp. 201-215. MR 2513338 (2010i:46086) 
[Cu-Li1] J. Cuntz and X. Li, The regular $C^{*}$-algebra of an integral domain, Quanta of maths, 149-170, Clay Math. Proc., 11, Amer. Math. Soc., Providence, RI, 2010. MR 2732050

[Cu-Li2] J. Cuntz and X. Li, $C^{*}$-algebras associated with integral domains and crossed products by actions on adele spaces, J. Noncommut. Geom. 5 (2011), no. 1, 1-37. MR2746649 (2011k:46093)

[La] M. Laca, From endomorphisms to automorphisms and back: dilations and full corners, J. London Math. Soc. (2) 61 (2000), no. 3, 893-904. MR.1766113 (2002a:46094)

[La-Ra1] M. Laca and I. Raeburn, Semigroup crossed products and the Toeplitz algebras of nonabelian groups, J. Funct. Anal. 139 (1996), no. 2, 415-440. MR1402771 (97h:46109)

[La-Ra2] M. Laca and I. Raeburn, Phase transition on the Toeplitz algebra of the affine semigroup over the natural numbers, Adv. Math. 225 (2010), no. 2, 643-688. MR 2671177

[Li] X. Li, Ring $C^{*}$-algebras, Math. Ann. 348 (2010), no. 4, 859-898. MR2721644

[Mur] G. J. Murphy, Crossed products of $C^{*}$-algebras by endomorphisms, Integral Equations Oper. Theory 24 (1996), no. 3, 298-319. MR1375977 (97f:46105)

[Sta] P. J. Stacey, Crossed products of $C^{*}$-algebras by *-endomorphisms, J. Austral. Math. Soc. Ser. A 54 (1993), 204-212. MR 1200792 (94a:46077)

Department of Mathematics, University of Oslo, P.O. Box 1053, Blindern, N-0316 OSLO, NORWAY

E-mail address: nadiasl@math.uio.no

Department of Mathematics, Westfälische Wilhelms-Universität Münster, EinsteinStrasse 62, 48149 Münster, Germany

E-mail address: xinli.math@uni-muenster.de 